\title{
COMMEMORATIVE LECTURE Neural mechanisms of cognitive memory
}

\author{
Yasushi Miyashita \\ Department of Physiology, The University of Tokyo School of Medicine, Tokyo, Japan
}

(Received for publication on March 5, 2004)

Key words: memory, vision, cognition, cerebral cortex, functional imaging

It is my great honor to be here for the award of the 2003 Keio Medical Science Prize. I express my gratitude to Keio University and Dr. Mitsunada Sakaguchi.

Knowledge or experiences are voluntarily recalled from memory by reactivation of their neural representations in the cerebral association cortex. Four questions are central in understanding this process: (1) Where in the brain is the mnemonic representation of objects localized? And how is it organized? (2) How is the representation created and what is the morphological and molecular basis of neural circuit reorganization? (3) Which neural circuits enable the reactivation of the representation in memory retrieval? (4) What is the neural basis of cognitive control in memory retrieval and in meta-memory judgment?

Clinical studies in humans have suggested that longterm declarative memory is stored in the neocortical association area that is also engaged in sensory perception (for review, see Miyashita, 1993, ${ }^{1}$ Miyashita \& Hayashi, $2000^{2}$ ). When electrical stimulation was applied via cortical surface electrodes placed on the temporal lobe during neurosurgery under local anesthesia, epileptic patients sometimes reported recollection of past perceptual experiences (Penfield \& Perot, 1963), ${ }^{3}$ suggesting that artificial electrical input to the putative memory storehouse might reactivate the "brain's record of auditory and visual experience". Recent experimental studies in humans and non-human primates moved beyond those classical clinical observations and revealed clearer and more solid views on the issues, particularly regarding the memory system of visual objects. In this lecture, I will first describe how memory engrams are organized in the cerebral cortex, focusing on the neural representation of semantically linked symbols/objects. I will then discuss how memory engrams are activated, highlighting two different retrieval processes, 'active' and 'automatic'. Finally, I will show you our recent attempts to clarify the neural basis of a metamemory system, which supervises the retrieval process and exerts cognitive control.

\section{Representation of Visual Objects: Organizing Memory Engrams}

Neuronal correlates of associative long-term memory were first reported in the monkey inferior temporal (IT) cortex by Miyashita (1988) ${ }^{4}$ and Sakai and Miyashita (1991). ${ }^{5}$ Their single-unit recordings identified two mnemonic properties of IT neurons: 1) that IT neurons can acquire stimulus selectivity through learning in adulthood; and 2) that their activity can link representations of temporally associated but geometrically unrelated stimuli. How these memory neurons function as basic elements of semantic networks, and how semantic networks are created through interaction among multiple representations in temporal cortical areas, is now firmly established (Miyashita and Chang, 1988, ${ }^{6}$ Miyashita and Hayashi, $2000^{2}$ ). In that regard, investigation of the neural basis of semantic networks has been greatly facilitated by reducing complex associative networks to elementary associative links between two objects, and then asking what are the neural mechanisms underlying such elementary associative links (Miyashita, 1993). ${ }^{1}$ The pair-association (PA) memory task is the best-known neuropsychological test with which to tap the memory of such an elementary pairwise associative relation (e.g. WMS-R, Wechsler, 1987). ${ }^{7}$ In the following section, we discuss how investigations using PA task revealed the neuronal machinery of associative memory in the IT cortex.

Dr. Miyashita is the recipient of the 2003 Keio Medical Science Prize.

Presented at the Keio Medical Science Prize Symposium, December 4, 2003.

Reprint requests to: Dr. Yasushi Miyashita, Department of Physiology, The University of Tokyo School of Medicine, 7-3-1, Hongo, Bunkyo-ku, Tokyo 113-0033, Japan 
A

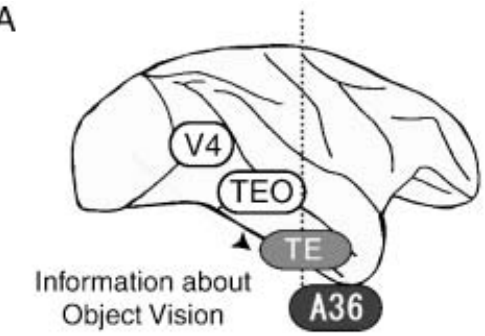

C

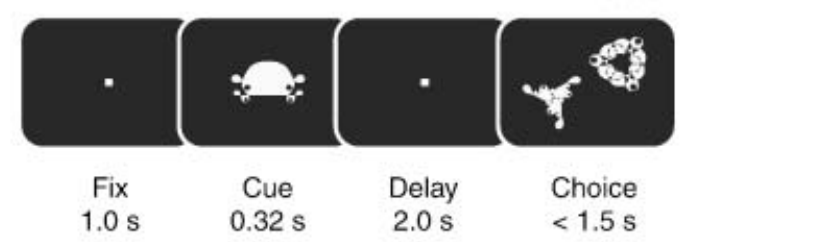

Fig. 1 A. Lateral view of a macaque brain. TE is located at the final processing stage of the ventral visual pathway. A36 is thought to be a part of the medial temporal lobe system. V4, visual area 4; TEO, area TEO. B. Coronal cross section indicated by the vertical line on the lateral view in panel $\mathrm{A}$. The black and gray areas indicate the locations of the recoding sites in TE and A36, respectively. C. Sequence of events in one trial of the PA task. Fixation points and cue stimuli were presented at the center of a video monitor. Choice stimuli were presented randomly in two of four positions on the video monitor.

\section{Forward processing of pair-association memory}

The IT cortex contains two cytoarchitectonically distinct but mutually interconnected areas: area TE (TE) and area 36 (A36) (Fig. 1A, 1B). TE is a unimodal neocortex and located at the final stage of the ventral visual pathway, which processes object vision (Miyashita, 1993). ${ }^{1}$ A36, on the other hand, is a limbic polymodal association area and a component of the medial temporal lobe memory system, which is involved in the formation of declarative memory (Higuchi and Miyashita, 1996). ${ }^{8}$ Naya et al. $(2003)^{9}$ found that association between the representations of different but semantically linked objects proceeds from TE to A36. To do this, they trained monkeys to perform the PA memory task in which meaningless computer-generated pictures were sorted randomly into pairs. We refer to each member of a pair as a paired associate. The monkeys were trained to memorize combinations of paired associates, which could not be predicted otherwise. In each trial of the task, a cue stimulus was presented, and the monkey was rewarded when he/she chose the paired associate of the cue (Fig. 1C). After training, extracellular spike discharges were recorded from single neurons in TE and A36.

A total of 2368 neurons were recorded from A36 (510 neurons) and TE (1858 neurons) in the three monkeys performing the PA task. Of those, 475 neu-
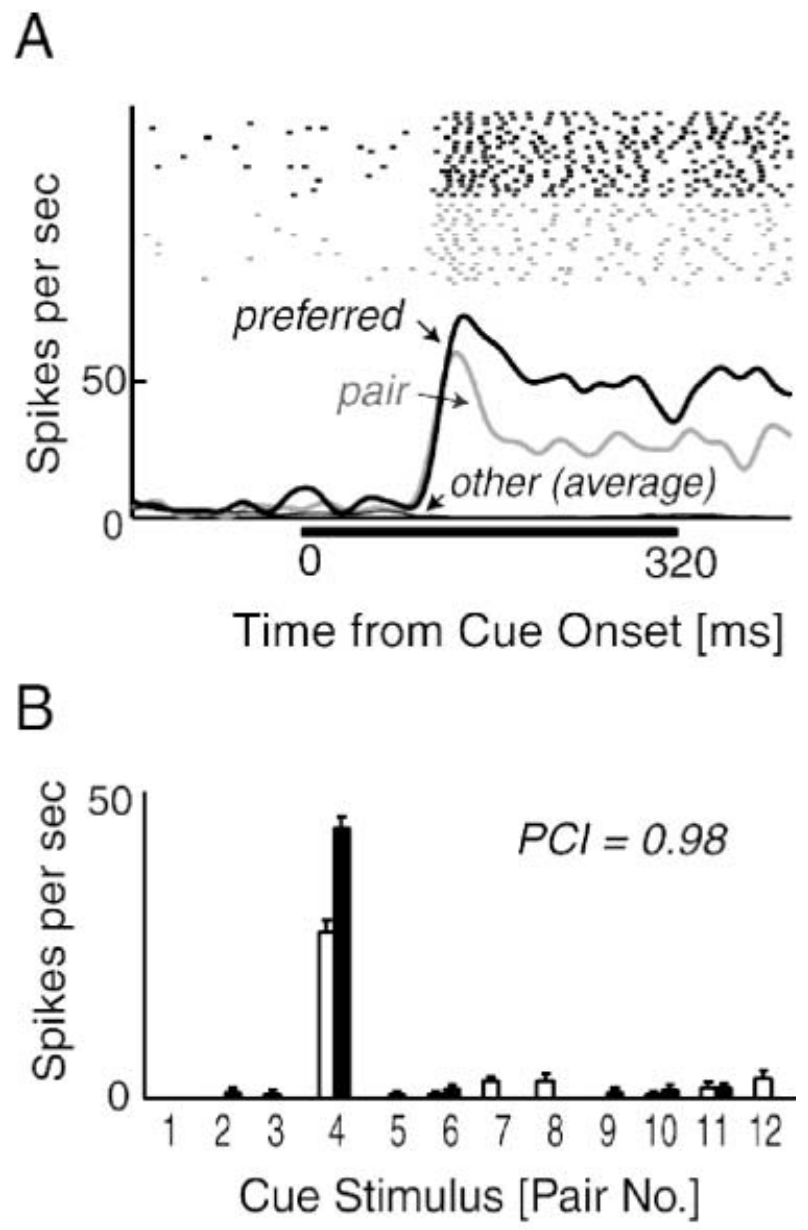

Fig. 2 Stimulus-selective responses to both paired associates. Data from a representative A36 neuron. A. Raster displays and PSTHs in trials where the preferred stimulus (preferred, thick black) or its paired associate (pair, thick gray) served as a cue. PSTH and the trials were temporally aligned at the cue onset. The black lines indicate responses to the preferred cue stimulus (preferred, thick black) or its paired associate (pair, thick gray). The thin black line denotes the averaged responses in the other trials (other). The horizontal gray bar indicates the cue presentation period. B. Mean discharge rates during the cue period (60-320 ms from the cue onset) for each cue presentation. Twelve pairs of stimuli are labeled on the abscissa. The open and filled bars in pair 1, for example, refer to the responses to stimulus 1 and 1', respectively. Error bars denote SEM. (Reproduce from Naya Y, et al: J Neurosci 2003; 23: 2861-2871, Copyright (C) (2003), with permission from Society for Neuroscience)

rons (85 neurons in A36 and 390 neurons in TE) showed responses to the cue presentation for at least one stimulus among the 24 learned stimuli (visually responsive cells). Out of them, 423 neurons (76 neurons in A36 and 347 neurons in TE) showed significant $(p<0.01$, ANOVA) stimulus selectivity during the cue period (60-320 ms from cue onset) (cue-selective cells). The responses of a representative cue-selective neuron in A36 are shown in Figures 2A and 2B. Note that one stimulus elicited the strongest response from this neu- 
ron during the cue period (Fig. 2A, thick black; Fig. $2 \mathrm{~B}$, filled bar in pair 4). This neuron was also activated when the paired associate of the preferred stimulus was presented (Fig. 2A, thick gray; Fig. 2B open bar in pair 4). In contrast to the robust responses to this stimulus pair, this neuron responded only negligibly when stimulus from any of the other pairs were presented as cue stimuli (Fig. 2A, thin black, the averaged responses to the other 22 stimuli; Fig. 2B). This type of neuron, which is found in the IT cortex and is referred to as a 'pair-coding neuron', selectively responds to both paired associates (pair-coding response). This property indicates that memory storage is organized such that single neurons can code both paired associates in the PA task.

The pair-coding responses in A36 with those in TE were compared by examining the distributions of the response amplitude for the pair trials. It was found that the distribution were significantly shifted toward positive values in both areas (A36, median $=0.27$; TE, median $=0.03 ; p<0.001$ in both areas, Wilcoxon's signed-rank test), with the distribution for the A36 neurons shifted to significantly higher values than that for the TE neurons $(p<0.001$, Kolmogorov-Smirnov test). Thus, in addition to the preferred stimulus, neurons in both A36 and TE responded selectively to the paired associate of the preferred stimulus, and the response was more prominent in A36 than in TE. In addition, the percentage of pair-coding neurons among the cue-selective neurons was significantly higher in A36 $(33 \%)$ than in TE $(4.9 \%)\left(p<0.001, \chi^{2}\right.$ test $)$ (Naya et al., 2003). ${ }^{9}$ This means that although neurons in both areas acquire stimulus selectivity through associative learning, the effect is engraved more intensely on the neuronal representation in A36 than in TE. The dramatic increase in the percentage of pair-coding neurons that one sees by going from TE to A36 indicates that the association between representations of paired associates proceeds forward through this anatomical hierarchy of the IT cortex.

\section{Circuit reorganization during formation of the pair- association memory}

It has been long hypothesized that memory engrams of declarative knowledge, as exemplified by the emergence of pair-coding neurons, develop with a structural and functional reorganization of neural circuits in the cerebral association cortices (Miyashita Y, 1993, ${ }^{1}$ Miyashita \& Hayashi, $2000^{2}$ ). This reorganization of neural circuits would be accomplished through a cellular program of gene expression leading to increased protein synthesis and then to alteration of synaptic connections. To date, this hypothetical framework has been primarily investigated in invertebrates and lower mammals, in which it is difficult to examine the organization of semantic memory. Still, the hypothesis as applied to semantic memory has been tested in a series of molecular biological studies carried out in monkeys (Tokuyama et al., 2000, ${ }^{10} 2002^{11}$ ), showing that upregulation of mRNA encoding proteins thought to be involved in structural reorganization occurred during formation of the pair-association memory. In this series of studies, the RT-PCR mRNA quantitation was combined with three unique experimental strategies. The first strategy entailed the use of split-brain monkeys, which were prepared by transecting the anterior commissure and the entire extent of the corpus callosum (Hasegawa et al., 1998). ${ }^{12}$ The fact that there was no interhemispheric transfer of mnemonic engrams in this preparation (Hasegawa et al., 1998) ${ }^{12}$ enabled us to compare mRNA expression between the left and right hemispheres within individual monkeys (Fig. 3A), thereby eliminating genetic and cognitive variations between individuals. The second strategy entailed the use of a visual discrimination (VD) task, rather than a notask condition, as the control (Fig. 3A). This enabled the motivational and attentional states in the two hemispheres, as well as the input of visual stimuli, to be appropriately balanced. The third strategy entailed training monkeys to first learn a 'rule' or 'strategy' component of the tasks using training stimulus sets, after which a test stimulus set was introduced for new learning of the declarative components of the task. Before the learning process with the test stimulus was complete, the animals were perfused, and expression of mRNA in the brains was evaluated. This enabled investigation of gene expression during formation of associative memory but not during formation of procedural memory.

Using the approach described above, it was found that expression of mRNA encoding Brain Derived Neurotrophic Factor (BDNF) was significantly higher in A36 of the PA hemisphere than in the VD hemisphere $(p<0.05)$ (Fig. 3B). In the early visual cortex (e.g., V1 or V4), by contrast, expression of BDNF mRNA did not differ in the two hemispheres (V1, $p>0.60 ; \mathrm{V} 4, p>0.87)$, indicating that the increased expression of BDNF mRNA level in A36 did not reflect a difference in the amount of visual input. The RT-PCR analysis also showed that expression of the mRNA encoding trkB, a specific receptor for BDNF, was slightly increased in A36 of the PA hemisphere, though the increase did not reach statistical significance. The expression of the mRNA encoding the immediate-early gene zif 268 was also selectively upregulated in A36 during formation of PA memory (Tokuyama et al., 2002). ${ }^{11}$ On the other hand, expression of a 'housekeeping gene', $\beta$-actin, did not differ between the two hemispheres in any of the cortical areas examined.

The spatial distribution of the BDNF mRNA was 

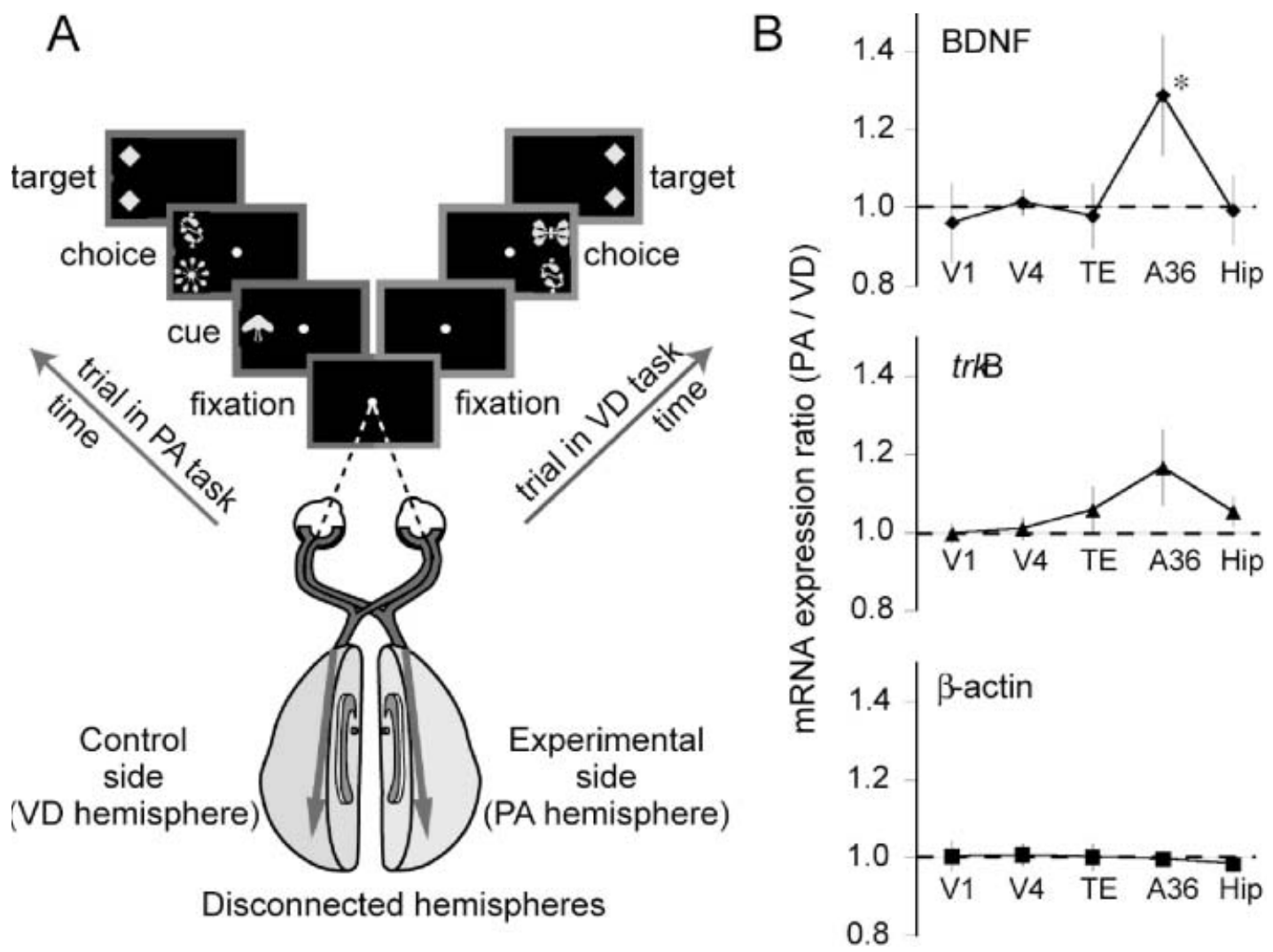

Fig. 3 A. Visual memory tasks for split-brain monkeys. Stimulus configuration for two visual memory tasks in different visual hemifields. Each split-brain monkey was trained to perform both a pair-association (PA) task and a visual discrimination (VD) task. During visual fixation, visual stimuli for the PA task were presented to one visual hemifield, while visual stimuli for the VD task were presented in the other hemifield. Using this configuration, split-brain monkeys did the PA task with one hemisphere (PA hemisphere) and the VD task with the other hemisphere (VD hemisphere). B. Intra-animal comparison of levels of expression of BDNF, trkB, and -actin mRNA in the PA and VD hemispheres. In each cortical area of each monkey, the mRNA expression ratio was calculated by dividing the level mRNA expression in the PA (experimental) hemisphere by that in the VD (control) hemisphere. The mRNA expression ratios were then averaged across animals and plotted for the indicated five cortical areas (mean \pm s.e.m.). Expression of BDNF mRNA was significantly higher in A36 of the PA hemisphere than A36 of the VD hemisphere. An asterisk indicates the significant difference between the PA and VD hemispheres $(p<0.05)$. (Reproduce from Tokuyama W, et al: Nat Neurosci 2000; 3: 1134-1142, Copyright (C) (2000), with permission from Society for Nature Publishing Group)

visualized using in situ hybridization (Fig. 4). Notably, BDNF mRNA-positive cells accumulated as a "patchy" cluster in A36 of the PA hemisphere, but not in the same area of the VD hemisphere, which suggests that upregulation of BDNF expression is associated with neurons located within the patches in A36. These patches were most prominent in layers $\mathrm{V} / \mathrm{VI}$, but were also observed in layers II/III (Fig. 4C), extending for at least $0.4 \mathrm{~mm}$ along the anterior-posterior axis. In contrast to the PA hemisphere, the VD hemisphere contained only scattered BDNF mRNA-positive cells in layers V/VI of A36 (Fig. 4B and D). And when the magnitude of the local increase of BDNF mRNA expression was estimated by grain-counting analysis (framed areas, Fig. 4A and B), it was found that significantly more neurons in the PA hemisphere expressed detectable levels of BDNF mRNA than in the VD hemisphere $[9.1 \pm 0.7 \%$ in the PA hemisphere vs. $3.7 \pm 0.6 \%$ in the VD hemisphere $\left(\chi^{2}=72.4\right.$, $p<0.001)]$. In area 35 of the PA hemisphere, expression of BDNF mRNA also seemed slightly stronger than in the VD hemisphere, but there were no differences in the patterns of BDNF mRNA expression in any other regions of the PA and VD hemispheres.

BDNF is thought to mediate activity-dependent synaptic plasticity, even in mature nervous systems. Consistent with that idea, its expression is regulated by changes in neuronal activity. Moreover, since zif 268 encodes a transcription factor, its expression could function as a trigger for a cascade of gene activation that leads to the cellular events underlying neuronal reorganization. Thus, analysis of the formation of PA memory has provided the first evidence supporting the hypothesis that BDNF contributes to the reorganization of neural networks, and that perhaps this reorganization is initiated by zif 268 , which triggers a cascade of gene activation. 

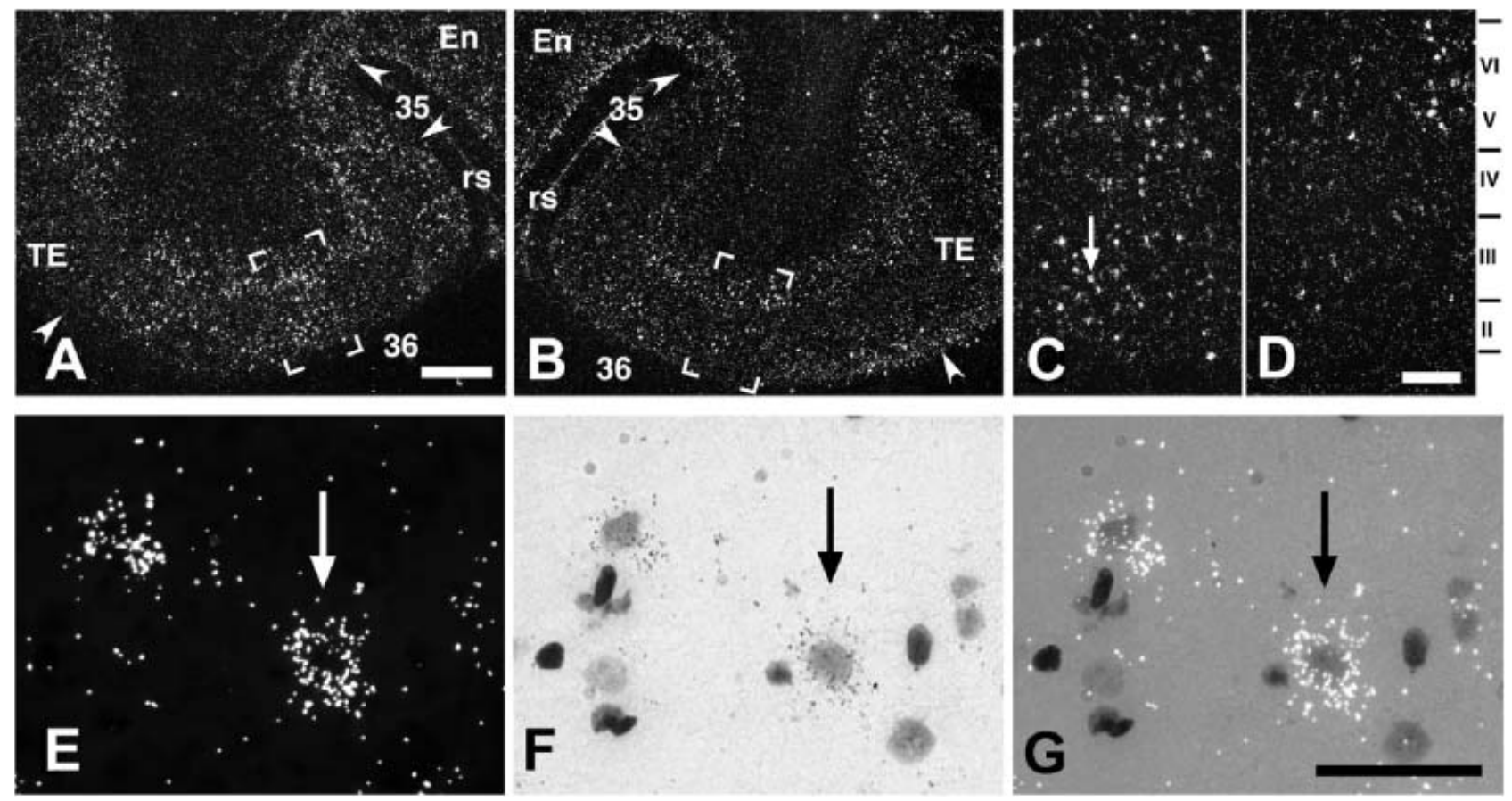

Fig. 4 In situ hybridization of BDNF mRNA. A-D. Distribution of BDNF mRNA in the IT gyrus of the PA (A) and VD (B) hemispheres. BDNF mRNA accumulated in a patch in A36 of the PA hemisphere (framed area), but not in A36 of the VD hemisphere. The framed areas in (A) and (B) are enlarged in (C) and (D), respectively. BDNF mRNA-positive cells were observed in layers V/VI and in layers II/III of the PA hemisphere $(\mathbf{C})$; the image of the cell marked by the arrow are defined in $(\mathbf{E}-\mathbf{G})$. En, entorhinal cortex; 35, area 35; 36, area 36; TE, area TE; rs, rhinal sulcus. Arrowheads mark the boundaries between different cortical areas. E-G. BDNF mRNA-positive cells in layers II/III of the PA hemisphere. The cell marked by an arrow in $(\mathbf{C})$ is enlarged and shown in darkfield (E), bringht field (F), and brightfield with epi-illumination (G). Silver grains were concentrated around lightly Nissl-stained neuronal nuclei. Cortical layers of A36 are indicated along margin of (D). Scale bars, $1 \mathrm{~mm}(\mathbf{A}, \mathbf{B}), 250 \mu \mathrm{m}(\mathbf{C}, \mathbf{D}), 50 \mu \mathrm{m}(\mathbf{E}-\mathbf{G})$. (Reproduce from Tokuyama W, et al: Nat Neurosci 2000; 3: 1134-1142, Copyright $(\mathrm{C}$ (2000), with permission from Society for Nature Publishing Group)

The location of the focal patch expressing BDNF approximates the location of aggregates of pair-coding neurons detected by single-unit recording (referred to as a 'hotspot') (Naya et al., 2003, ${ }^{9}$ Yoshida et al., 2003 ${ }^{13}$ ). A combined anatomical-physiological analysis recently showed that structural reorganization does indeed occur at hotspots and that the fiber terminals of pictureselective neurons in TE are retracted from out of the hotspot in A36 but remain to project within the hotspot (Yoshida et al., 2003). ${ }^{13}$ We therefore suggest that BDNF expression may induce axonal and synaptic reorganization in the hotspot in A36, and that such reorganization of local networks is detected electrophysiologically as a change in neuronal stimulus selectivity, typically as the emergence of pair-coding neurons.

\section{Activation of Memory Engrams: 'Active' vs. 'Automatic' Retrieval}

There appears to be two types of memory retrieval processes, automatic and active. You know that sometimes one needs no effort to recall and at other times one has to strive toward a successful recall. We refer to the former as automatic retrieval and to the latter as active retrieval (Miyashita \& Hayashi, 2000, ${ }^{2}$ Petrides, $2000^{14}$ ). In the previous section, we discussed visual associative memory stored in the IT cortex. It follows that such long-term memory could then be retrieved from the IT cortex by either of these two processes. In this section, we will suggest that whether retrieval is automatic or active depends on whether the retrieval signal is created within the network of the IT cortex or runs from the prefrontal (PF) cortex to the IT cortex. We begin by introducing a study that showed that the automatic memory retrieval signal flows backward through the IT cortex, from A36 to TE.

\section{Automatic retrieval signal: backward spread of memory retrieval signal in the inferior temporal cortex}

The theory of semantic network visualizes a retrieval of an item as activation of a corresponding node at the network. The neural correlate of such a node-activation was first reported in the pair-association task by Sakai and Miyashita (1991). ${ }^{5}$ They found a group of IT neurons that showed an activation related to the retrieval of the paired associates from a cue stimulus. The response is referred to as pair-recall response. Using a 
A

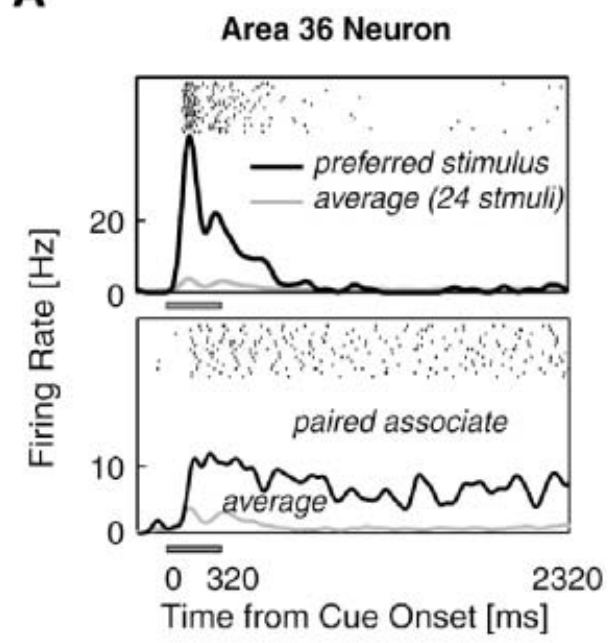

C

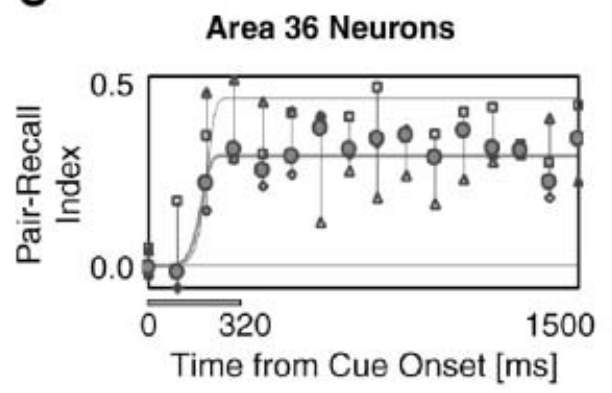

B

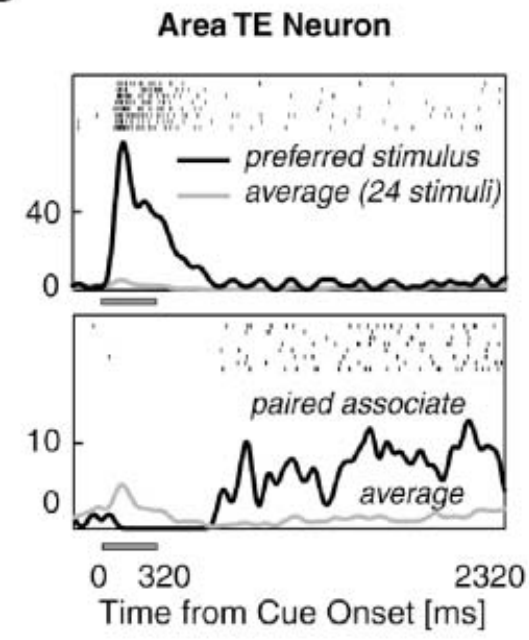

D

Fig. 5 Neuronal activity related to memory retrieval, as shown by a single cell in A36 (A) and in TE (B). For the raster displays [(A) and (B)] and PSTH, responses were temporally aligned at the cue onset in trials where the preferred stimulus (upper panel) or its paired associate (lower panel) served as a cue. The black lines indicate responses to the preferred cue stimulus (upper panel) or its paired associate (lower panel); the gray lines indicate mean responses to all 24 stimuli. (C and D) Temporal dynamics of averaged PRI $(t)$ for the population of stimulus-selective neurons (A36, $\mathrm{n}=45$; TE, $\mathrm{n}=69$ ). Mean values of PRI $(t)$ were plotted every $100 \mathrm{~ms}$ for A36 (C) and TE (D) neurons (filled circle, total; open diamond, monkey 1; open square, monkey 2; open triangle, monkey 3). Thick lines [in (C) and (D), respectively] indicate the best-fit Weibull functions for the population-averaged $\operatorname{PRI}(t)$ in the two areas. Thin lines indicate the same but for the neurons whose $\operatorname{PRI}(t)$ increased above the 5\% significance level. (Reproduce from Naya Y, et al: Science 2001; 291: 661-664, Erratum in 2001; 291: 1703, Copyright (C) (2001), with permission from Society for the American Association for the Advancement of Science)

modified PA task (PA with a color switch task), Naya et al. $(1996)^{15}$ showed that this pair-recall response indeed corresponded to the recall of the visual image in subject's mind, since IT neurons started to fire just after a color switch that signaled the necessity and timing of memory retrieval during its delay period and the IT neurons also stopped to fire just after another color switch that signaled retrieval of other memorized items. Such change of neuronal discharge did not occur when a color switch signaled a simple maintenance of shortterm memory. Therefore, this type of delay activity in the IT cortex represents the internal target that is retrieved from long-term memory. Recently, it was reported that this target-related activity is transmitted backward, from A36 to TE, as illustrated below (Naya et al., 2001). ${ }^{16}$

The representative responses of a TE neuron and an
A36 neuron showing the target-related activity specified by a cue stimulus (Sakai and Miyashita, 1991, ${ }^{5}$ Naya et al., 1996 $6^{15}$ ) are shown in Figure 5A and 5B. One stimulus elicited the strongest response during the cue period and the response continued into the delay period (upper panel). In the trial when the paired associate of this preferred stimulus was presented as a cue, the neurons in both A36 and TE exhibited the highest delay activity among the stimuli (lower panel). This type of activity is referred to as target-related. The onset of the target-related activity of the TE neuron was later than that of the A36 neuron (lower panel).

The time course of the target-related delay activity of each neuron was examined by considering the responses to all cue stimuli. The partial correlation coefficients of instantaneous firing rates at time $t$ for each cue stimulus were calculated with the visual 
A Bottom-up condition
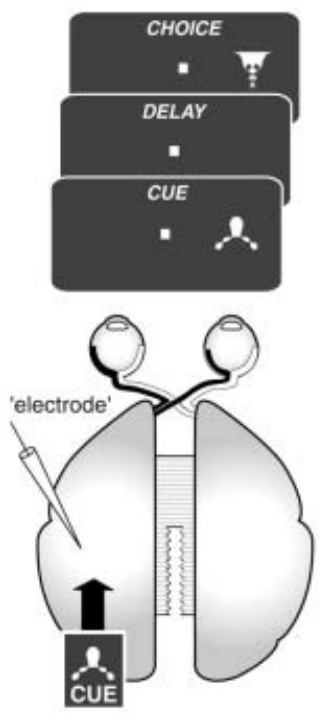

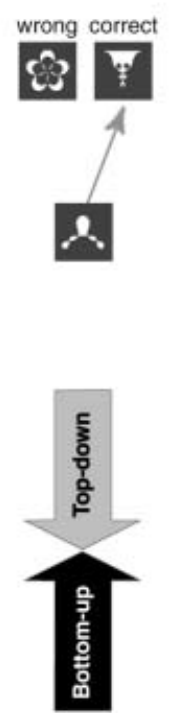

B Top-down condition
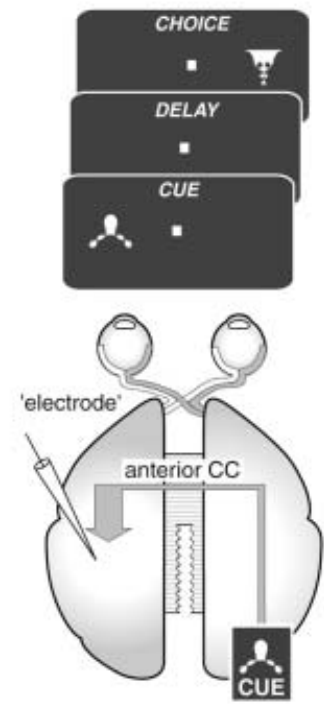

Fig. 6 The posterior-split paradigm. A. Bottom-up condition. Visual stimuli (cue and choice pictures) were presented in the hemifield contralateral to the recording site ('electrode') in the IT cortex. The monkeys were trained to memorize visual stimulus-stimulus associations among 20 cue and 5 choice pictures. In each trial, while the monkey held a lever and maintained fixation, cue and choice pictures were sequentially presented parafoveally with a delay. If the choice picture was the correct one associated with the cue, the monkey had to release the lever. Bottom-up sensory signals (black arrow) would be detected in this condition. B. Top-down condition. The protocol is the same as in A, but the cue was presented in the hemifield ipsilateral to the recording site; the choice was presented contralaterally. In posterior-split-brain monkeys, the sensory signal does not reach visual areas in the opposite hemisphere. In this condition, only top-down signals (gray arrow) could activate IT neurons through feedback connections from the prefrontal cortex.

responses to its paired associate (pair-recall index, PRI). The time courses of the average $\operatorname{PRI}(t)$ across the population of stimulus-selective neurons significantly differed in A36 and TE (repeated-measures ANOVA, $p<0.0001$ ) (Fig. 5C, 5D). The $\operatorname{PRI}(t)$ for the A36 neurons began to increase during the cue period and developed with a rapid time course. The $\operatorname{PRI}(t)$ for the TE neurons, by contrast, increased slowly and reached a plateau in the middle of the delay period. To summarize this section, memory-retrieval signals appeared first in A36, after which TE neurons were gradually recruited to represent the sought target. Thus mnemonic information that was extracted from longterm storage, spread backward, from A36 to TE.

Top-down signaling appears when active retrieval is required

A clinical case study helps us to highlight the active retrieval in humans and provides a clue to an experi- mental model with which to investigate active retrieval (Sidtis et al., 1981). ${ }^{17}$ In that study, an epileptic patient who had undergone posterior callosotomy was given a word in his left visual field. He could never read the name of it directly, although he claimed to 'see' its image in his mind. He was eventually able to answer the name using inferential strategies based on his mental image. His limited ability suggests that his right hemisphere was transmitting to his left hemisphere semantic information about the stimulus but not the actual stimulus. After the callosum was completely sectioned, semantic information was no longer transferred from his right hemisphere to left. Hasegawa et al. $(1998)^{12}$ combined this posterior-split-brain paradigm with the associative memory task in monkeys. In the posterior-split-brain monkey, in which the posterior corpus callosum and the anterior commissure are sectioned, the cortex receives bottom-up visual information only from the contralateral visual field (Fig. 6A). With this paradigm, long-term memory acquired through stimulus-stimulus association does not transfer interhemispherically via the anterior corpus callosum; nonetheless, when the visual cue was presented to one hemisphere, the anterior callosum could instruct the other hemisphere to retrieve the correct stimulus specified by the cue. Thus, although visual long-term memory is stored in the temporal cortex, memory retrieval is under the executive control of the PF cortex.

Direct proof of the existence of top-down signal from the PF cortex to the temporal cortex and of its contribution to the active retrieval process was provided by single-unit recordings from the posterior-split-brain preparation (Tomita et al., 1999). ${ }^{18}$ In this protocol, inferior temporal neurons in one hemisphere ('electrode', Fig. 6A) are to be activated by bottom-up visual inputs when an object is presented in the visual hemifield contralateral to the recording site. When the object is presented in the ipsilateral hemifield, however, these neurons should not be able to receive bottom-up visual inputs. Any neural activation should therefore reflect top-down inputs from the PF cortex (Fig. 6B). It was found that a considerable number of IT neurons did indeed receive top-down signals from the PF cortex; the activity of one such IT neuron is shown in Figure 7. This neuron was not only activated by contralateral presentation of stimuli (Fig. 7, bottom-up response, black), but was also activated by ipsilateral presentation of stimuli (Fig. 7, top-down response, gray). In these neurons, which showed stimulus-selectivity in both top-down and bottom-up responses, it was found that the latency was significantly longer in the top-down condition $(p<$ 0.001 ). These top-down responses were abolished after transection of the remaining anterior corpus callosum. The partial split-brain studies in human and monkeys reveal the events occurring during the active retrieval 


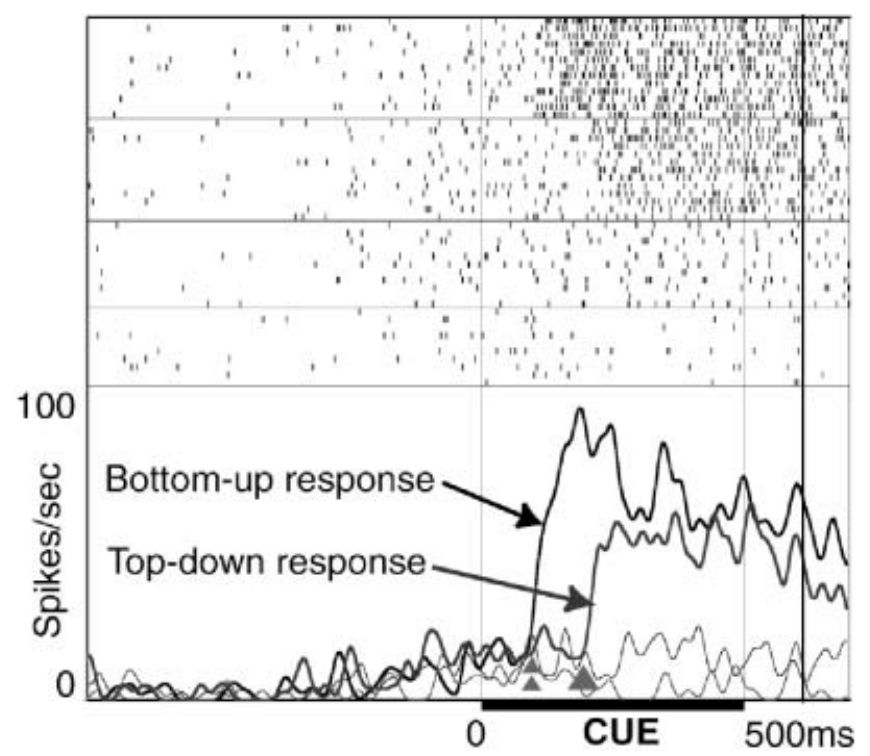

Fig. 7 Activity of a single IT cell in the top-down condition (topdown, gray; bottom-up, black). Raster displays and PSTH were temporally aligned at the cue onset. In the PSTH, thick lines show responses to the preferred cue, whereas thin lines show responses to a null cue. The onset of the top-down response (arrowhead) was later than that of the bottom-up response (double arrowhead). (Reproduce from Tomita H, et al: Nature 1999; 401: 699-703, Copyright C (1999), with permission from Society for Nature Publishing Group)

process, which are indicative of purely top-down signaling.

Imaging studies carried out in humans have further confirmed that the PF cortex play a key role in the active retrieval process. Activation of the PF cortex during memory retrieval is widely observed in functional neuro-imaging studies employing a variety of psychological paradigms and test modalities, including recognition tests, word-stem tasks, word-fragment tasks, paired associates tasks, free recall and recency judgment (Miyashita \& Hayashi, 2000, ${ }^{2}$ Cadret et al., $2001,{ }^{19}$ Fletcher et al., 1998, ${ }^{20}$ Konishi et al., 2002, ${ }^{21}$ Wagner et al., $1998^{22}$ ). Among these studies, Fletcher et al. $(1998)^{20}$ found that the right dorsal PF cortex (BA9/46) is strongly activated when monitoring demands were emphasized, while the right ventral PF cortex (BA45) showed greater activation when external cueing was emphasized. Wagner et al. $(1998)^{22}$ suggested that right $\mathrm{PF}$ activation reflect retrieval attempt including initiation of retrieval search or evaluation of the products of retrieval. In addition, Cadret et al. $(2001)^{19}$ attempted to identify more focal areas that were specifically related to active retrieval by matching the retrieval and control conditions in terms of depth of encoding, decision-making and postretrieval monitoring. They found activity related to active retrieval to be selectively increased within area 47/12 in the ventrolateral PF cortex. Earlier anatomical studies showed that area $47 / 12$ is strongly connected with the inferotemporal cortical region. Thus we suggest that the source of the top-down signaling reported by Tomita et al. (1999) ${ }^{18}$ may be a homologue of BA47/12 (see also Ohbayashi et al., 2003). ${ }^{23}$ The process of active retrieval could be involved where memory traces are accessed through inferential strategies, either implicit or explicit, which then raises the question of how such inferential processes are cognitively controlled.

\section{Metamemory: Cognitive Control of Memory System}

In daily life, you might have experienced a situation in which, although you were unable to answer a question in a free recall, you were sure you could have answered correctly if it was a multiple-choice question. In fact, you accessed related items in your semantic network, since there is a positive correlation between the objective score in the recognition test and the degree of a subjective feeling whether you knew the answer or not. Metamemory refers to knowledge about one's memory capabilities and knowledge about strategies that can aid memory (Kikyo, Ohki \& Miyashita, 2002). ${ }^{24}$ Metamemory requires execution of extensive retrieval process and, at the same time, supervises the retrieval process. Kikyo et al. (2002) ${ }^{24}$ successfully identified brain areas related to a metamemory system in humans using a "feeling-of-knowing" (FOK) paradigm, which is a well-established tool for assessing metamemory system (Fig. 8A). The FOK is a subjective sense of knowing a word before recalling it - i.e., a sense that "I know that I know it". Based on the recalljudgment-recognition (RJR) paradigm, subjects in this experiment were asked to recall word answers for general-information questions during fMRI scans. After the scans, the subjects wrote their answers to the recalled questions and were instructed to judge their degree of FOK to the non-recalled questions on a three-point scale, where $3=\langle\mathrm{I}$ definitely could recall the answer if given hints or more time $\rangle ; 2=\langle$ I probably would recognize the answer $\rangle ; 1=\langle$ I definitely did not know $\rangle$. Event-related fMRI with a parametric analysis showed stronger activity in the bilateral IFGs (BA 47), left MFG (BA 46/9, BA 10) and ACC/SMA (BA 32/24/6) when people have a greater FOK (Fig. $8 \mathrm{~B})$. These activation areas are referred to here as the FOK regions. Among these FOK regions, subregions in the bilateral IFGs (BA 47) were not recruited for successful recall processes, suggesting a specific role of these regions in human metamemory system. One of the FOK regions was located in the anterior portion of the left MFG, BA10. This area was regarded as a part of the memory areas in the anterior prefrontal cortex (AFC) in some literatures and was related to retrieval strategy and/or "third level of executive control". 

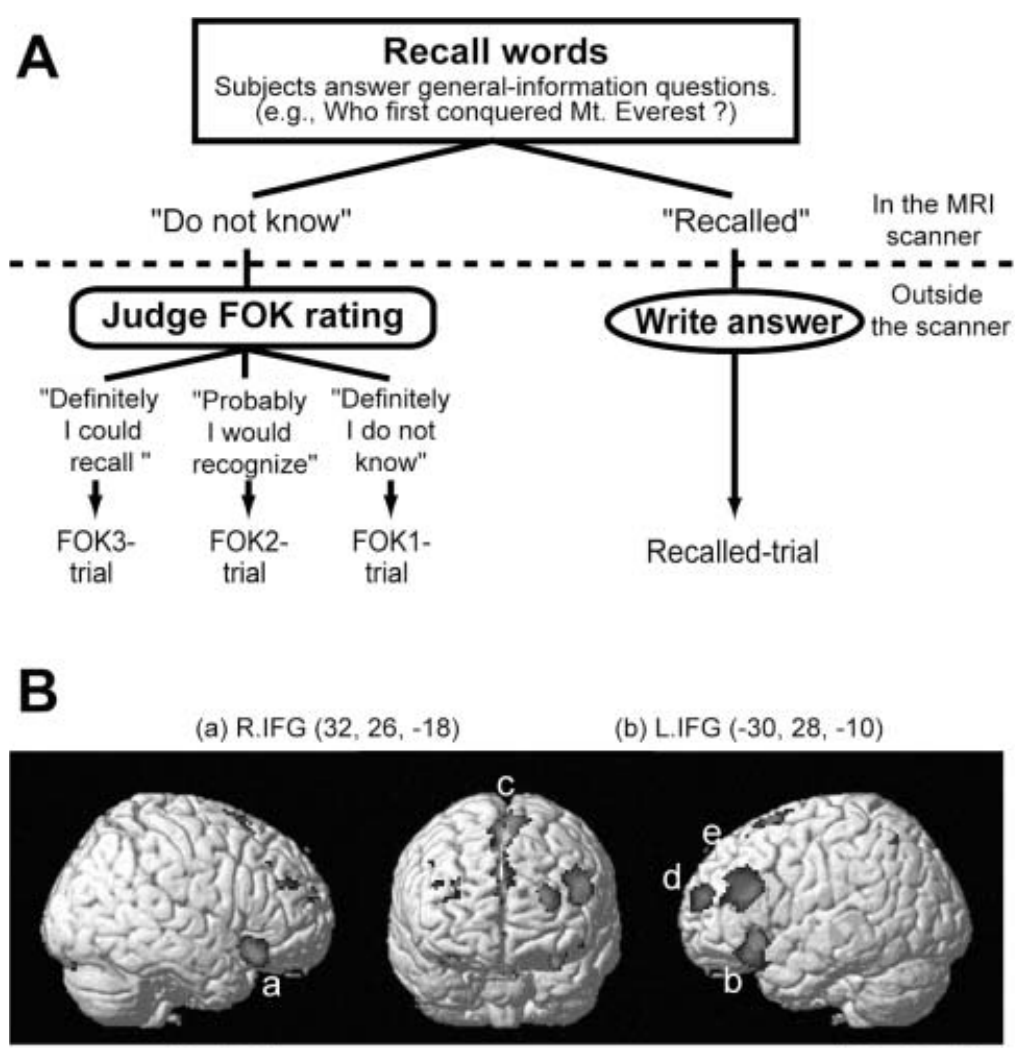

(c) $\operatorname{ACC}(-2,26,36)$

(d) L.MFG $(-28,60,18)$

(e) L.MFG $(-50,26,26)$

Fig. 8 A. Experimental procedures of the Feeling-of-Knowing (FOK) trials. Subjects were required to recall word answers to general-information questions during fMRI scans. By pressing buttons, they indicated whether they recalled the target words or not. Then, outside the scanner, they judged their degree of FOK to the nonrecalled questions on a scale of three for the non-recalled questions, or they wrote their answers to the recalled questions. Each trial was sorted into trial type (Recalled, FOK3, FOK2, and FOK1) according to the participant's judgment and was subjected to event-related fMRI analysis. B. Regions involved in FOK and/or successful recall. A subset of the FOK regions was also activated in successful recall (c, d, and e). A subset of the FOK regions in the bilateral IFGs was not activated in successful recall (a and b). A small subset of the FOK region in the left IFG was also activated in successful recall, but its cluster size did not reach the significant, given the correction for the whole brain multiple comparisons. (Reproduce from Kikyo H, et al: Neuron 2002; 36: 177-186, Copyright $\odot$ (2002), with permission from Society for Elsevier Science)

\section{Future Perspective}

The extensive investigations described in this chapter have been revealing a whole picture of the semantic memory system. There are, however, still pieces missing that will be needed to make the picture complete. For example, in the metamemory system, how are the active retrieval subsystem and its cognitive control subsystem integrated?; How does each component of the identified distributed metamemory network in the PF cortex differentially support those subsystems?; Is there any specific molecular/cellular basis for those differential functional subsystems? It is anticipated that a number of missing pieces will be found when the gap between the information provided by invasive studies carried out with monkeys and that provided by non-invasive human imaging studies is filled (Miyashita and Hayashi, 2000). ${ }^{2}$ Most of the detailed knowledge of the anatomy, function and cellular basis of the cortex has come from studies in monkeys (Miyashita, 1993). ${ }^{1}$ With that as background, using the same methods to study humans and monkeys would advance our understanding of the neural organization of higher order cognitive function. For example, fMRI is a method that may bridge this gap by enabling direct comparison of the functional organization of the brains of monkeys and humans. Using that approach, Nakahara et al. (2001) ${ }^{25}$ observed that, when subjects performed a high-level cognitive task, transient activation related to cognitive set shifting occurred in focal regions of the prefrontal cortex in both monkeys and humans, and that these functional homologues were located in cytoarchitectonically equivalent regions in the posterior part of ventrolateral prefrontal cortex. Recently, we successfully extended these comparative neuroimaging studies into a higher spatial resolution one with a high-field (4.7T) MRI system 
(Koyama et al., 2004). ${ }^{26}$ Such comparative imaging between humans and macaques has the potential to provide significant new insight into the evolution of cognition in primates.

Finally, I hope these integrative approaches facilitate further advancement of systems neuroscience regarding cognition and also encourage young students and postdoctoral students who have just started their scientific career towards the investigation of the human mind.

Acknowledgements: I would like to gratefully acknowledge the help of my collaborators, Kuniyoshi Sakai, Hiroyuki Okuno, Wataru Tokuyama, Yuji Naya, Kiyoshi Nakahara and Isao Hasegawa. Many more students than I can name individually have my grateful thanks and acknowledgement for their assistance in these researches.

\section{References}

1. Miyashita Y: Inferior temporal cortex: where visual perception meets memory. Annu Rev Neurosci 1993; 16: 245-263

2. Miyashita Y, Hayashi T: Neural representation of visual objects: encoding and top-down activation. Curr Opin Neurobiol 2000; 10: $187-194$

3. Penfield WP, Perot P: The brain's record of auditory and visual experience. A final summary and discussion. Brain 1963; 86: 595-696

4. Miyashita Y: Neuronal correlate of visual associative long-term memory in the primate temporal cortex. Nature 1988; 335: 817820

5. Sakai K, Miyashita Y: Neural organization for the long-term memory of paired associates. Nature 1991; 354: 152-155

6. Miyashita Y, Chang HS: Neuronal correlate of pictorial shortterm memory in the primate temporal cortex. Nature 1988; 331: $68-70$

7. Wechsler D: Wechsler Memory Scale-Revised, San Antonio, The Psychological Corporation, Harcourt Brace Jovanovich, 1987

8. Higuchi S, Miyashita Y: Formation of mnemonic neuronal responses to visual paired associates in inferotemporal cortex is impaired by perirhinal and entorhinal lesions. Proc Natl Acad Sci USA 1996; 93: 739-743

9. Naya Y, Yoshida M, Miyashita Y: Forward processing of longterm associative memory in monkey inferotemporal cortex. J Neurosci 2003; 23: 2861-2871

10. Tokuyama W, Okuno H, Hashimoto T, Xin Li Y, Miyashita Y: BDNF upregulation during declarative memory formation in monkey inferior temporal cortex. Nat Neurosci 2000; 3: 11341142

11. Tokuyama W, Okuno H, Hashimoto T, Li YX, Miyashita Y:
Selective zif 268 mRNA induction in the perirhinal cortex of macaque monkeys during formation of visual pair-association memory. J Neurochem 2002; 81: 60-70

12. Hasegawa I, Fukushima T, Ihara T, Miyashita Y: Callosal window between prefrontal cortices: cognitive interaction to retrieve long-term memory. Science 1998; 281: 814-818

13. Yoshida M, Naya Y, Miyashita Y: Anatomical organization of forward fiber projections from area TE to perirhinal neurons representing visual long-term memory in monkeys. Proc Natl Acad Sci USA 2003; 100: 4257-4262

14. Petrides M: Frontal lobes and memory. In: Boller F, Grafman J, eds, Handbook of Neuropsychology, Amsterdam, Elsevier, 2000; pp. 67-84

15. Naya Y, Sakai K, Miyashita Y: Activity of primate inferotemporal neurons related to a sought target in pair-association task. Proc Natl Acad Sci USA 1996; 93: 2664-2669

16. Naya $\mathrm{Y}$, Yoshida M, Miyashita $\mathrm{Y}$ : Backward spreading of memory-retrieval signal in the primate temporal cortex. Science 2001; 291: 661-664 Erratum in: Science 2001; 291: 1703

17. Sidtis JJ, Volpe BT, Holtzman JD, Wilson DH, Gazzaniga MS: Cognitive interaction after staged callosal section: evidence for transfer of semantic activation. Science 1981; 212: 344-346

18. Tomita H, Ohbayashi M, Nakahara K, Hasegawa I, Miyashita Y: Top-down signal from prefrontal cortex in executive control of memory retrieval. Nature 1999; 401: 699-703

19. Cadoret G, Pike GB, Petrides M: Selective activation of the ventrolateral prefrontal cortex in the human brain during active retrieval processing. Eur J Neurosci 2001; 14: 1164-1170

20. Fletcher PC, Henson RN: Frontal lobes and human memory: insights from functional neuroimaging. Brain 2001; 124: 849881

21. Konishi S, Uchida I, Okuaki T, Machida T, Shirouzu I, Miyashita Y: Neural correlates of recency judgment. J Neurosci 2002; 22: 9549-9555

22. Wagner AD, Desmond JE, Glover GH, Gabrieli JD: Prefrontal cortex and recognition memory. Functional-MRI evidence for context-dependent retrieval processes. Brain 1998; 121: 19852002

23. Ohbayashi M, Ohki K, Miyashita Y: Conversion of working memory to motor sequence in the monkey premotor cortex. Science 2003; 301: 233-236

24. Kikyo H, Ohki K, Miyashita Y: Neural correlates for feeling-ofknowing: an fMRI parametric analysis. Neuron 2002; 36: $177-$ 186

25. Nakahara K, Hayashi T, Konishi S, Miyashita Y: Functional MRI of macaque monkeys performing a cognitive set-shifting task. Science 2002; 295: 1532-1536

26. Koyama M, Hasegawa I, Osada T, Adachi Y, Nakahara K, Miyashita Y: Functional magnetic resonance imaging of macaque monkeys performing visually guided saccade tasks; comparison of cortical eye fields with humans. Neuron 2004; 41: 795 807 